\title{
RECIDIVA PÉLVICA DE TUMOR UROTELIAL, TRAS CISTOPROSTATECTOMÍA RADICAL CON NEOVEJIGA ORTOTÓPICA
}

\author{
D. PASCUAL REGUEIRO, E. MALLÉN MATEO, M.A. TRÍVEZ BONED, \\ M.A. GARCÍA GARCÍA, Ma..J. GIL SANZ, L.A. RIOJA SANZ
}

Servicio de Urología. Hospital Universitario Miguel Servet. Zaragoza.

Actas Urol Esp. 27 (8): 629-632, 2003

\section{RESUMEN \\ RECIDIVA PÉLVICA DE TUMOR UROTELIAL, TRAS CISTOPROSTATECTOMÍA RADICAL CON NEOVEJIGA ORTOTÓPICA}

Presentamos un caso de recurrencia pelviana de un tumor urotelial, cinco meses después de la cistoprostatectomía radical.

El estudio patológico de los fragmentos de resección transuretral no demostró afectación de la grasa.

El tratamiento fue cistoprostatectomía radical con neovejiga ileal tipo Hautmann.

El estudio patológico de la pieza quirúrgica reveló infiltración de la grasa en varios puntos pero con uretra y ganglios linfáticos sin afectación.

PALABRAS CLAVE: Neovejiga ileal ortotópica. Recidiva tumoral.

\section{ABSTRACT}

UROTELIAL TUMOR PELVIC RECURRENCE AFTER RADICAL CYSTOPROSTATECTOMY AND ORTOTHOPIC NEOBLADDER

We present a case of an urotelial tumor pelvic recurrence, five months after radical cystoprostatectomy. No fat infiltration demonstrated in the pathological study of the transurethral resection pieces.

The treatment was a radical cystoprostatectomy with an ileal ortothopic Hautmann type neo-bladder.

The pathological study of the chirurgical piece demonstrated fat infiltration in some points but urethra and lymphatic nodes free.

KEY WORDS: Ortothopic ileal neo-bladder. Tumor recurrence.

$\mathrm{L}^{\infty}$ os tumores uroteliales vesicales, presentan una historia natural caracterizada por su tendencia a la progresión y diseminación, tanto local como a distancia, así como a la recidiva en cualquier punto de la vía urinaria tratándose de una enfermedad difusa del urotelio.

El tratamiento de estos tumores, se encuentra bastante estandarizado, siendo conservador el destinado para los superficiales, mediante resecciones endoscópicas, mientras que para los tumores infiltrantes está indicado el tratamiento radical con sustitución vesical o derivación urinaria posterior, en dependencia de la situación general del paciente.

En el caso de afectación tumoral más allá de las capas musculares: grasa perivesical, invasión de seminales en el varón, o de órganos vecinos, la cistectomía radical no está indicada salvo con carácter paliativo, ya que la incidencia de recidiva y progresión tumoral es muy alta, a pesar de realizar terapias adyuvantes como son la quimioterapia sistémica o la radioterapia pélvica. 
Sin embargo es la anatomía de los fragmentos de una resección endoscópica, lo que nos llevan en la mayoría de los casos a indicar la cistectomía, y en algunas ocasiones, el análisis posterior de la pieza quirúrgica, demuestra una invasión mayor de lo sospechado, ensombreciendo el pronóstico, disminuyendo notablemente la supervivencia y favoreciendo la recidiva y progresión tumoral.

\section{CASO CLÍNICO}

Se trata de un paciente de 57 años de edad, con múltiples antecedentes a destacar:

Diabetes mellitus insulino dependiente $\mathrm{y}$ fumador compulsivo, llegando a consumir entre 3 y 4 paquetes diarios.

La conjunción de ambos factores determina una retinopatía diabética, una cardiopatía isquémica severa y una isquemia crónica de extremidades inferiores.

Esta patologia de base condiciona un tratamiento de base con antiagregantes y antagonistas de la vitamina $\mathrm{K}$, y múltiples intervenciones:

- Diciembre $1998 \rightarrow$ by-pass aortocoronario.

- Enero $1999 \rightarrow$ endarterectomía ileofemoral con by-pass.

- Marzo $1999 \rightarrow$ colocación de endoprótesis aórtica lumbar y amputación del 5o dedo del pie derecho.

- Abril $1999 \rightarrow$ amputación transmetatarsiana del pie izquierdo.

El paciente consulta por haber presentado un episodio de hematuria macroscópica monosintomática que se había repetido de forma caprichosa y autolimitada.

La exploración resulta totalmente anodina, con un tacto rectal I, no sospechoso.

Se solicita un análisis general y un PSA, que resultan normales.

Asímismo se realiza una ecografía genitourinaria, en la que se aprecia un tracto urinario superior normal, y una tumoración vesical vegetante de unos $17 \mathrm{~mm}$ en cara lateral derecha.

Se lleva a cabo una resección endoscópica del citado tumor, hallándose una uretra normal, ambos meatos uretrales libres, y una tumoración de aspecto infiltrante de unos 3-4 cm que abarca gran parte de la cara lateral derecha. El estudio anatomopatológico de la lesión demuestra una invasión de las capas musculares profundas, sin afectación de la grasa perivesical, con biopsia del lecho de la resección y uretral negativas, y alto grado de indiferenciación $\rightarrow$ pT2b G3.

Se realiza así mismo estudio de diseminación, con un rastreo óseo negativo, y una TAC en la que no se evidencia afectación hepática ni ganglionar (Fig. 1).

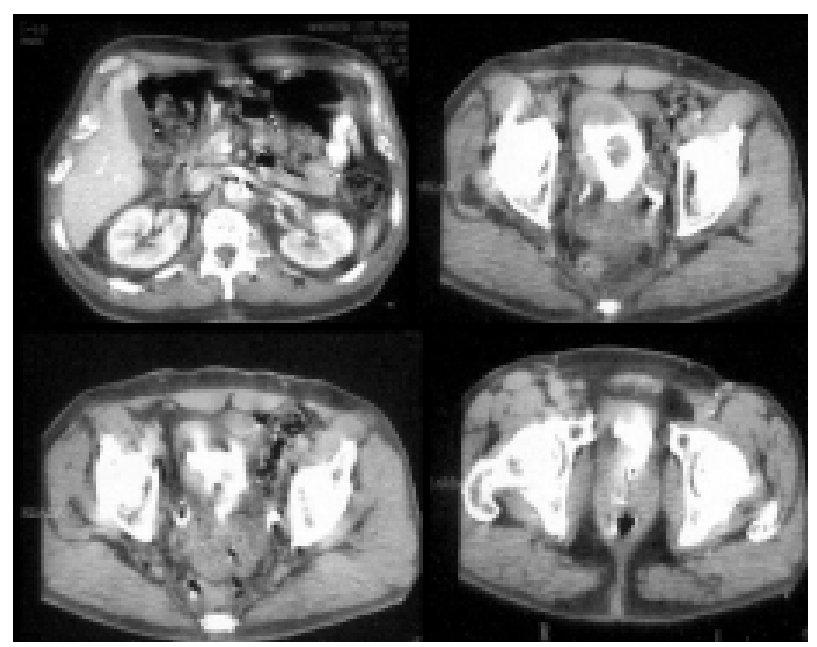

FIGURA 1. Imágenes del TAC realizado previo a la cirugía. Captación y eliminación bilateral de contraste por ambos riñones sin presentar ectasia pielocalicilar. A nivel vesical se observa engrosamiento difuso, con crecimiento endoluminal que afecta a la práctica totalidad de la cara vesical derecha, con aspecto infiltrante.

Ante estos hallazgos, se lleva a cabo una cistoprostatectomía radical, con sustitución vesical según técnica de Hautmann, con chimenea antirreflujo de Studer, y con anastomosis uretero neovesical tipo Wallace II, trascurriendo el postoperatorio con normalidad siendo dado de alta a los 21 días de la intervención.

El estudio anatomopatológico de la pieza revela un carcinoma transicional sólido, poco diferenciado y ulcerado que infiltra todas las capas musculares y que afecta focalmente el tejido adiposo con invasión perineural en dos puntos. Los extremos quirúrgicos, la próstata y los fragmentos de uréteres terminales se encuentran libres de afectación, así como 9 ganglios linfáticos iliacos izquierdos y 4 derechos $\rightarrow$ pT3a G3 p53 (-).

Dado que le paciente se encuentra asintomático, y dado su grave trasfondo cardiológico y vascular, se desestima de entrada el tratamiento quimioterápica adyuvante. 
Cuatro meses tras la intervención, acude a urgencias, por presentar un cuadro de hematuria de una semana de evolución, acompañado de dolor en fosa renal derecha.

La analítica muestra una leve acidosis metabólica que es tratada con bicarbonato y una insuficiencia renal con cifras de creatinina plasmática de $2,8 \mathrm{mg} / \mathrm{dl}$.

Asimismo se comprueba una ectasia renal derecha grado II.

Ante estos hallazgos se coloca una nefrostomía percutánea derecha, realizándose opacificación a través de la misma. Se comprueba paso hasta uréter pelviano con un afilamiento terminal del uréter derecho a nivel de la anastomosis uretero-neovesical. En estos momentos se sospecha una estenosis de dicha anastomosis, o una recidiva tumoral a ese nivel (Fig. 2).

Por la persistencia del sangrado, se realiza una revisión endoscópica , encontrando una uretra normal, una neovejiga con coágulos en su interior pero sin neoformaciones. Se aprecia salida de orina hematúrica a través de la chimenea de Studer. Clínicamente el paciente mantiene creatininas plasmáticas en torno a $2 \mathrm{mg} / \mathrm{dl}$, con correcto funcionamiento de la nefrostomía.

Se produce un empeoramiento del estado general, con dolor importante en la extremidad derecha y periné.

Una semana después se realiza TAC abdomino-pélvico con los siguientes hallazgos:

- Moderada ectasia bilateral.

- Indemnidad de la neovejiga.

- Dilatación de la arteria femoral derecha.

- Voluminosa masa tumoral compatible con recidiva pélvica, a la derecha de la neovejiga, extendiéndose a lo largo de la pared pélvica derecha y hacia escotadura ciática, con aspecto infiltrante, y con un tamaño aproximado de 70 x $53 \mathrm{~mm}$. Afectación del músculo obturador interno y piramidal derechos (Fig. 3).

Se decide intentar tratamiento quimioterápico con fines paliativos, para intentar disminuir el volumen tumoral y controlar el sangrado.

Por este motivo se realiza nefrostomía izquierda, consiguiendo mejoría de la función renal, con creatininas plasmáticas de $1,5 \mathrm{mg} / \mathrm{dl}$.

Se inicia el primer ciclo de quimioterapia con carboplatino y vinblastina, (dado el estado del

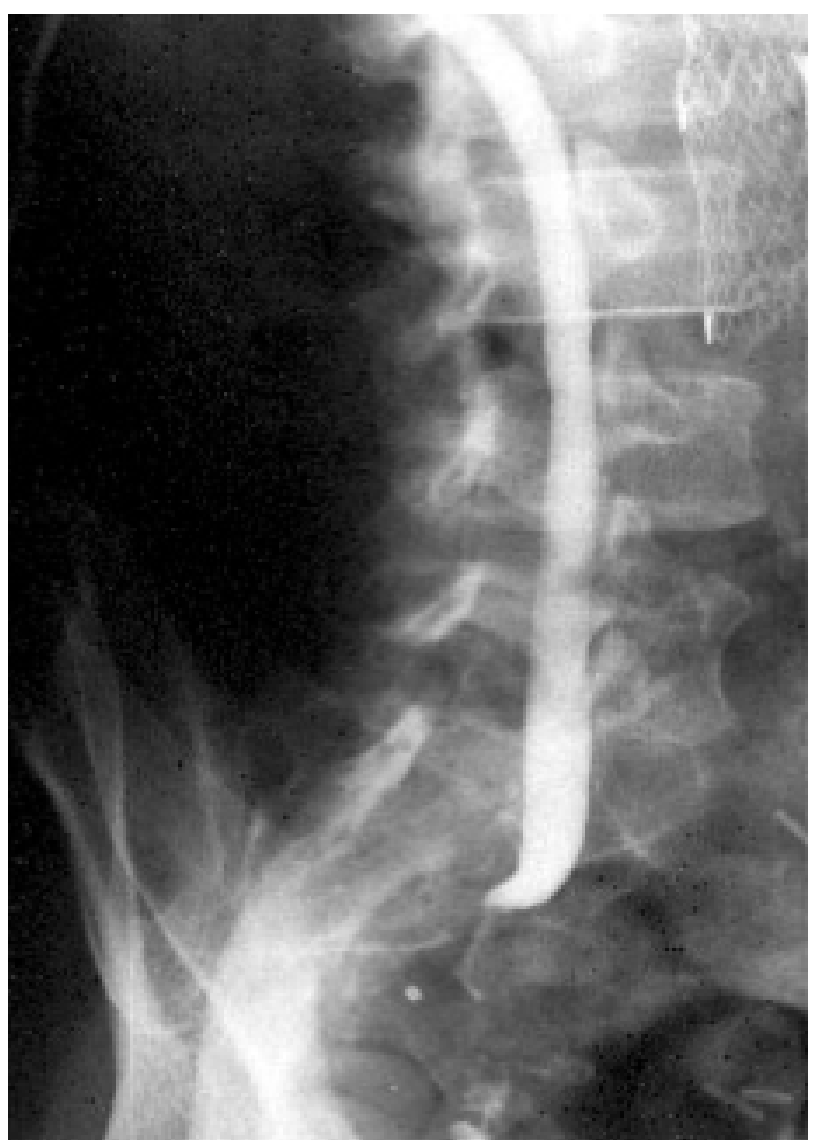

FIGURA 2. Opacificación realizada tras la colocación de la primera nefrostomia, en la que se evidencia una dilatación importante de todo el sistema derecho, con un afilamiento de su porción distal que permite un paso filiforme de contraste hacia neovejiga.

paciente se rehusó el tratamiento con ciclos de CMV, más agresivo, que es el estandarizado en nuestro servicio).

El paciente sufrió un deterioro progresivo de su estado general falleciendo tres meses después.

\section{DISCUSIÓN}

Las pautas de actuación y tratamiento de los tumores uroteliales vesicales, son bien conocidas, y se encuentran bastante estandarizadas y aceptadas.

En el caso de demostrarse la afectación de las capas musculares de la pared vesical, el tratamiento con resecciones endoscópicas pierde su validez con intención curativa, y queda relegado a pacientes con sintomatología, que por edad o patología concomitante, no se encuentren en condiciones de ser sometidos a una cirugía radical. 


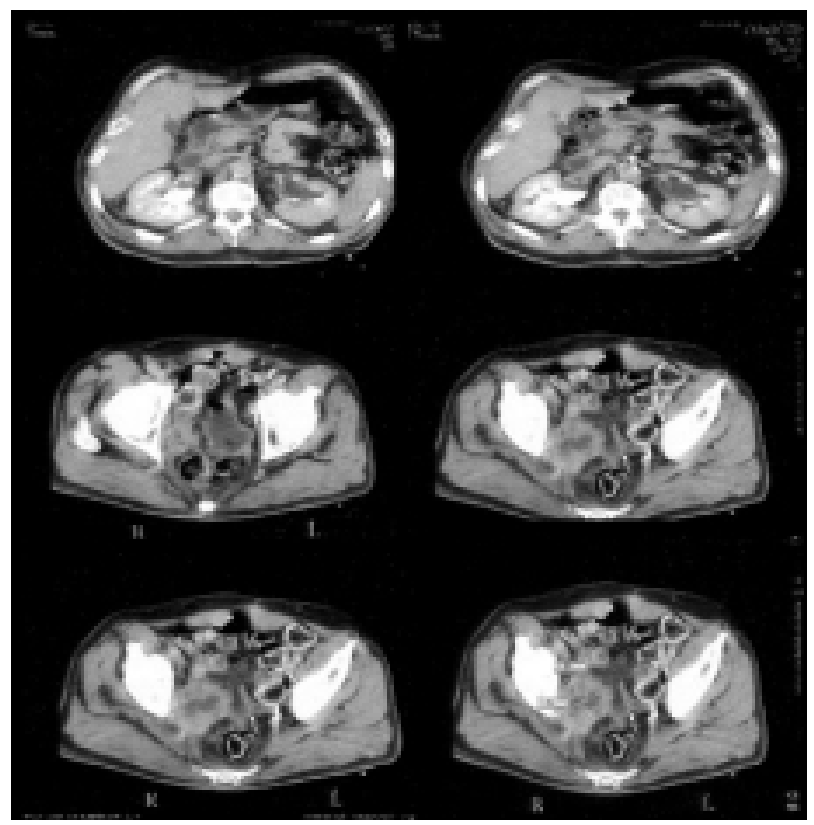

FIGURA 3. Imágenes del TAC realizado cinco meses después de la cirugia radical. Dilatación importante pielocalicilar derecha. Gran masa tumoral que capta contraste de forma heterogénea, que se extiende a lo largo de la cara lateral derecha de la pelvis, que desplaza e infiltra la neovejiga.

También es cierto que la indiferenciación tumoral y el grado de invasión de la pared vesical y de estructuras adyacentes, determinan un peor pronóstico y una mayor incidencia de progresión y de recidiva ${ }^{3,4}$.

Esto hace que la cirugía radical, cuando la invasión tumoral afecta a grasa o estructuras adyacentes vesicales, pierda su intención curativa y se realice sólo en algunos casos, y con fines paliativos.

Si bien es verdad que el estudio anatomopatológico de los fragmentos de resección, y el estudio de diseminación tumoral, apoyado en TAC, ECO y rastreo óseo, nos permiten una aproximación diagnóstica bastante fiable y por consiguiente una indicación de cirugía con bastantes garantías, la visión del campo quirúrgico y/o el posterior análisis de la pieza quirúrgica, pueden revelar una mayor afectación tumoral que la esperada, una vez ya realizada la cirugía radical.

En estos casos el pronóstico queda ensombrecido, haciendo que la incidencia de recidiva y progresión local y a distancia del tumor sea altísimas, como así refleja la literatura.

El Dr. Hautmann ${ }^{1}$, presentó una serie de 347 pacientes en los cuales se produjo recurrencia tumoral en 43, con un tiempo medio de 10 meses. 17 de ellos presentaron conjuntamente metástasis a distancia y actualmente han fallecido 36 de ellos por la enfermedad, 3 por complicaciones derivadas de la quimioterapia y uno por otro motivo, con una supervivencia media de 17 meses.

En nuestro caso se indicó tratamiento radical con un estudio de diseminación negativo, un estudio anatomopatológico de los fragmentos de resección con afectación únicamente de los planos musculares, y al que se le realizó una cistectomía y reconstrucción vesical ortotópica con intención curativa, y que posteriormente el estudio de la pieza quirúrgica reveló afectación de la grasa perivesical.

Desestimada en un principio la quimioterapia por la enfermedad de base, la historia natural de la enfermedad, determina la aparición a los cinco meses de la cirugía de una recidiva tumoral a nivel de la pelvis con infiltración de la neovejiga sin posibilidad de tratamiento ${ }^{2}$.

Esto ha determinado que una indicación, basada en un estudio completo de la enfermedad, planteada con una intención curativa, y sin presentar hallazgos relevantes en el acto quirúrgico, quede marcada en su evolución por un mayor grado de afectación tumoral que el sospechado, determinando una progresión y evolución fatal de la misma.

\section{REFERENCIAS}

1. HAUTMANN RE, SIMON J.: Ileal neobladder and local recurrence of bladder cancer: patterns of failure and impact on function in men. J Urol 1999 dec; 162 (6): 1963-1966.

2. TEFILLI MV, GHEILER EL, TIGUERT R, GRIGNON DJ, FORMAN JD, PONTES JE,WOOD DP Jr: Urinary diversión related outcome in patients with pelvic recurrence after radical cystectomy for bladder cancer. Urology 1999 may; 53 (5): 999-1004.

3. FREEMAN JA,TARTER TA,ESRIG D, STEIN JP, ELMAJIAN DA, CHEN SC, GROSHEN S, LIESKOVSKY G, SKINNER DG.: Urethral recurrence in patients with orthotopic ileal neo bladders. J Urol 1996 nov; 156 (5): 1615-1619.

4. JONES J, MELCHIOR SW, GILLITZER R, FICHNER J, EL-MEKRESH M, THUROFF JW.: Urethral recurrence of transitional cell carcinoma in a female patient after cystectomy and orthotopic ileal neobladder. J Urol 2000 nov; 164 (5): 1646.

Dr. D. Pascual Regueiro

Avda. Compromiso de Caspe, 27-29, $2^{\circ}$ A

50002 Zaragoza

(Trabajo recibido el 16 noviembre de 2001) 